\title{
Conventional and Digital Workflow Planning for Maxillary Teeth Restoration with Porcelain Laminate Veneers: A Clinical Report
}

\author{
Shatha Alshali (iD)', Rayan Asali \\ 'Prosthodontics Department, Faculty of Dentistry, King Abdulaziz University, Jeddah, 21589, Saudi Arabia; ${ }^{2}$ Prosthodontics Department, King Fahad \\ Armed Forces Hospital, Jeddah, Saudi Arabia
}

Correspondence: Shatha Alshali, Prosthodontics Department, Faculty of Dentistry, King Abdulaziz University, Idara St, P.O. Box 80200, Jeddah, 2I589, Saudi Arabia, Tel +966 5648I I876, Email alshali.shatha@gmail.com

\begin{abstract}
Nowadays, media-influenced patients have the desire to "extremely rapidly" alter their teeth to "ideal" and "too bright" ones. Although some esthetically demanding cases need to be thoroughly planned and treated through prolonged phases, other cases can be handled quickly. In such cases, we can witness the impact of many computer software programs for digital smile design that have penetrated clinical practice. This clinical report describes a conventional and digital step-by-step treatment workflow for restoring anterior maxillary teeth with porcelain laminate veneers. Two digital smile design software (GPS and Nemo DSD 3D) were utilized and compared with the conventional workflow. Feldspathic veneers were chosen for their superior optical properties. Case complexity and operator's experience played a significant role in the selection between the conventional and digital workflow. The advanced digital programs, while having many advantages, are not necessarily superior to the traditional technique. The digital smile design programs used in this case demonstrated an effective and predictable workflow. They can achieve comprehensive digital smile design with a satisfactory esthetic outcome, yet the conventional workflow gave conservative and esthetically pleasing results.
\end{abstract}

Keywords: feldspathic porcelain, veneers, digital smile design, digital workflow

\section{Introduction}

Nowadays, media-driven expectations inspire the desire for "ideal" and "extremely white" teeth in a rapidly accomplished procedure. ${ }^{1}$ To comply with such requests, we witness now the impact of many digital smile design computer programs that have been introduced to clinical practice. ${ }^{1}$ Digital smile design programs are multi-purpose conceptual tools that can reinforce the diagnostic vision, improve communication, and enrich treatment outcome by allowing detailed analysis of the patient's facial and dental characteristics. ${ }^{2,3}$ Smile Design Pro, Planmeca Romexis Smile Design, Nemo Digital Smile Design 3D system (DSD), and Guided Positioning System (GPS) are some examples of such systems. Various image editing instruments enable the operator to close gaps, graft tooth structure segments, adjust the smile line, and change the teeth shade within the patient's photographs or scanned models. Teeth can be selected from a large digital library, and their position, rotation, and shape can be modified to construct the perfect smile design for the patient. Once established in the software, it can then be converted into either a conventional or a virtual diagnostic waxup replica to simplify subsequent clinical procedures. ${ }^{4,5}$

GPS is a 2D smile design software, developed by Dr. Alain Methot (Wilmington, DE, USA), in which the design can be manually converted to conventional wax-up after calibrating the stone cast with the patient's photographs. ${ }^{4}$ However, data transfer from the virtual design to the laboratory stone model is difficult and susceptible to errors. ${ }^{6,7}$ The DSD system (Nemotec, Madrid, Spain) was developed to analyze and digitally design a smile. It is a minimally invasive digitalized process, spanning from preoperative image acquisition to the computer-aided design and computer-aided manufacturing ( $\mathrm{CAD} / \mathrm{CAM}$ )-printed mockup. The mockup can be precisely fabricated from an approved virtual 
diagnostic waxing, without additional conversion. ${ }^{4}$ This system can thus drastically reduce operator errors that are usually associated with the conventional manual steps, and improve the accuracy and reproducibility of the prosthetic procedure. $^{7}$

The conventional technique is based on a planning process after clinical then radiological examination, intra- and extra-oral analysis, occlusal evaluation, and impressions to fabricate study casts. ${ }^{7,8}$ In this technique, the dental technician fabricates a diagnostic wax-up replica based on established tooth proportion guidelines and the treatment objectives. The wax-up can also be used to fabricate provisional restorations. This technique depends to a great extent on the technician's hand skills, experience, and ability to follow the guideline. However, it has a high probability for human errors to occur. ${ }^{7,8}$

The most used indirect materials are feldspathic ceramics and lithium disilicate. Feldspathic ceramics well known of their superior esthetics, color stability, higher translucency and biocompatibility. As for lithium disilicate ceramics, in addition to the esthetics and functional properties, it showed greater fracture resistance in comparison to feldspathic ceramics, enhancing their clinical longevity. Regarding esthetics characteristics, both are available in a wide shade range, ability to imitate dental fluorescence and translucency, both can assure color and brightness stability. ${ }^{9}$ The fitness of the final crown and the design of tooth preparation play an important role in the success of these restorations. ${ }^{10}$

This clinical report aims to describe a conventional and digital treatment workflow for restoring maxillary teeth with porcelain laminate veneers. Two different digital smile design software (GPS and DSD) were utilized and compared with the conventional workflow.

\section{Clinical Report}

A 40-year-old female with good general health was referred to the Prosthodontics Department at the School of Dentistry in King Abdulaziz University, Jeddah, Saudi Arabia. A written informed consent has been provided by the patient to have the case details and any accompanying images published. This clinical report did not require an approval from the institutional review board. Her chief complaint was the loss of anterior teeth structure caused by sleep bruxism.

At the first appointment, primary impression casts were made, and a series of facial and intraoral images were taken after receiving the participant's approval. The patient had an average smile line, showing ten teeth when smiling. Her teeth had a triangular shape, and the buccal corridors were absent. The canine cusp tips and incisal edges of the maxillary incisors were lost but the patient had a stable occlusion and there was no need to increase the occlusal vertical dimension (Figure 1).

The patient also had a defective ceramo-metal crown on tooth \#25. She was diagnosed with dental attrition that caused the loss of incisal teeth structure of about $1.5 \mathrm{~mm}$ and an open margin crown on tooth \#25. A treatment plan for maxillary teeth restoration from tooth $\# 15$ to $\# 25$ was proposed, aiming at the most natural appearance that could

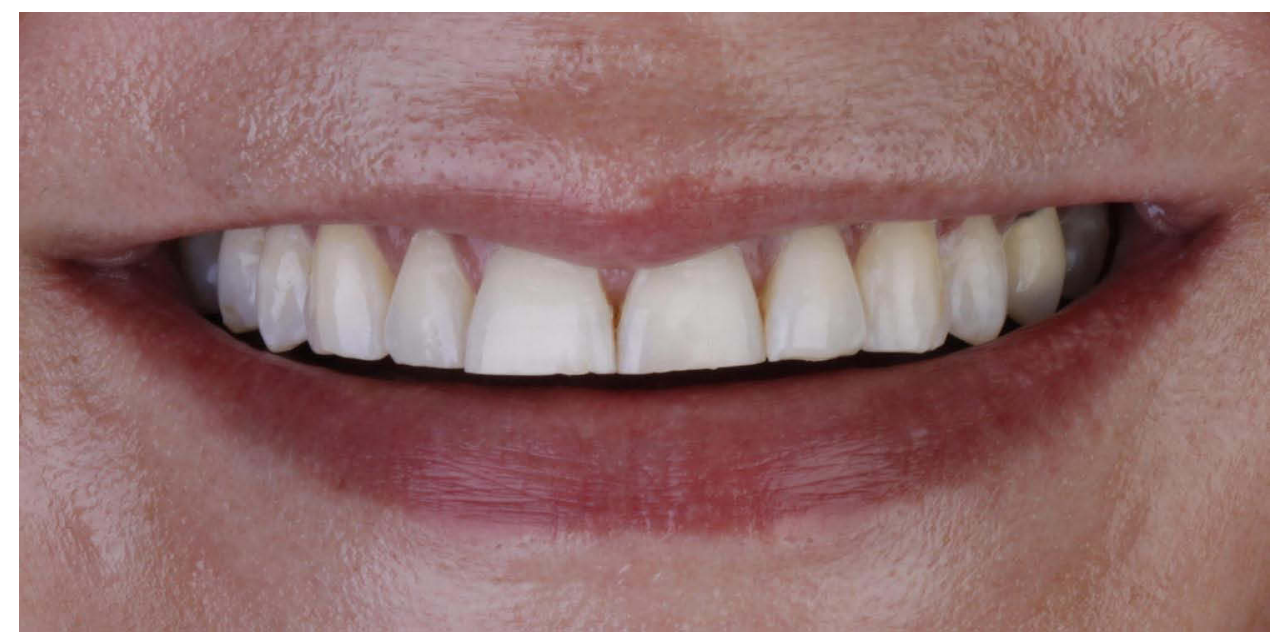

Figure I The patient's pretreatment smile. Note the dental attrition. 


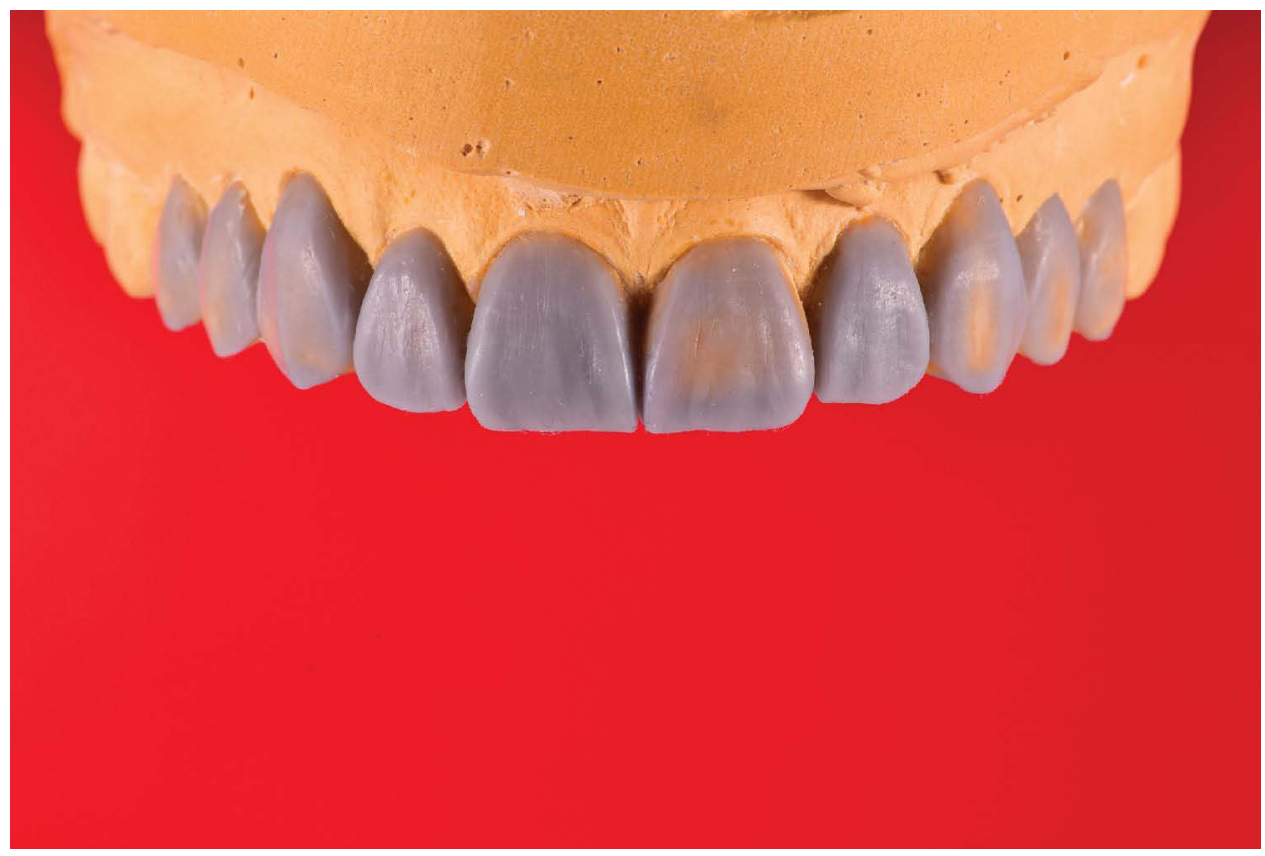

Figure 2 Conventional diagnostic wax-up.

complement her face and lips. The plan was to elongate the maxillary incisors by $1.5 \mathrm{~mm}$ and restore the canine cusp tips and incisal embrasures. In a labiopalatal direction, a position halfway between the most protruded and retruded central incisors was chosen for the final restorations. The treatment plan was augmented by analysis using different digital smile design systems. The objective was to compare two digital smile design systems (GPS and DSD) and the conventional workflow in selecting a treatment proposal.

\section{Workflow}

For the conventional workflow, a dental technician fabricated a diagnostic wax-up replica based on established tooth proportion guidelines, the patient's images, and the treatment objectives (Figure 2).

Frontal full face and close-up smile images were acquired and imported into the GPS software. Horizontal calibration was done. The images were standardized by the scaling and rotation tools on the horizontal and vertical reference guides (Figure 3).

A digital mock-up was designed following the guidelines. These guidelines were then transferred to the stone cast, using a digital ruler that was calibrated by measuring the central incisor size. A guided wax-up was then completed on the stone cast. Using a silicone index, the design was tested by transforming the wax-up into a preparation guide. It was also used to fabricate the provisional restorations (Figure 4).

For the DSD workflow, maxillary and mandibular casts were scanned by an inEos X5 scanner (Dentsply Sirona Dental System, Rome, Italy). The horizontal reference plane and the facial midline were determined by Digital Facebow and then transferred to the scanned cast by overlapping it with the photos. The dimensions of the photos were matched by using two vertical lines on the distal aspects of the canines as references (Figure 5A). Three-dimensional smile analysis was done, and a digital mock-up was designed on the screen, following the proportions and symmetry guidelines (Figure 5B). Two final 3D designs were printed; one for a mock-up that followed the most protruded tooth (Figure 6A); the other for a provisional restoration, mimicking the final design (Figure 6B). The trial restorations were fabricated by using a silicone impression material (Hydrorise Putty, Zhermack SpA, Badia Polesine, Italy) over the printed cast from the DSD (Figure 7A) and the conventional wax-up (Figure 7B). The indices were placed over the teeth with bis-acrylic resin then esthetics and phonetics were evaluated and approved. The GPS mock-up was digitally presented and compared 


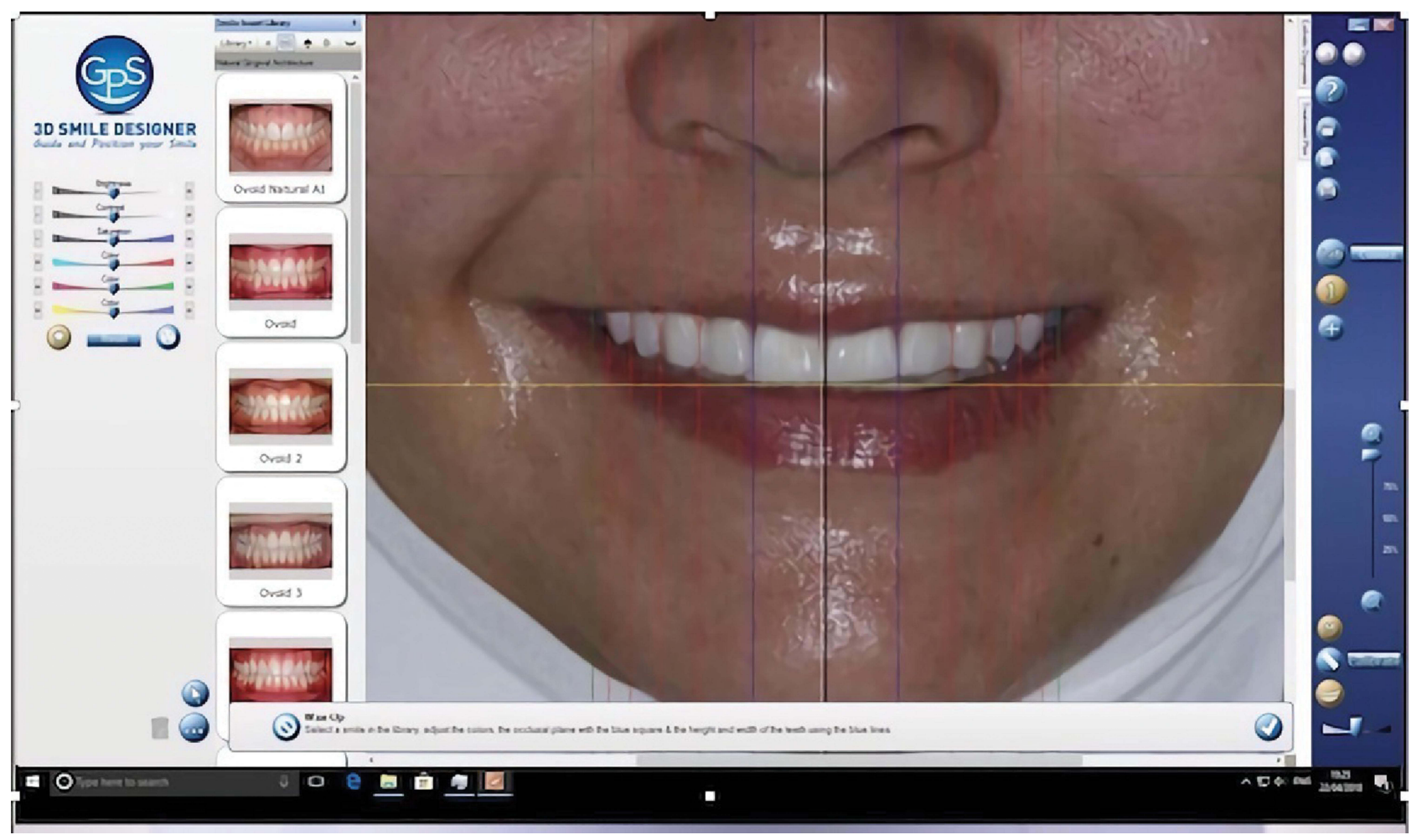

Figure 3 Guided positioning system (GPS) smile image calibration.

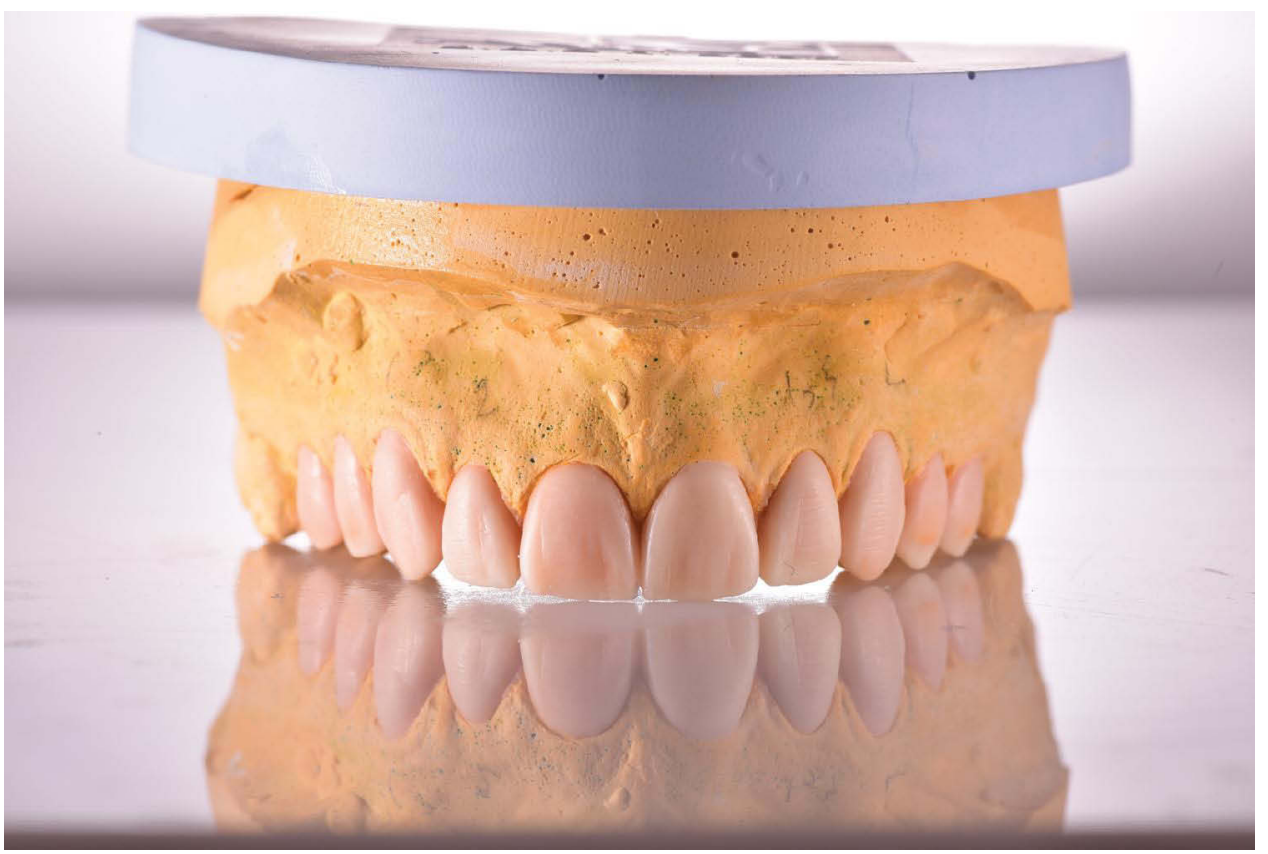

Figure 4 Guided positioning system (GPS) guided wax-up. Digital mock-up was transferred to stone cast after calibration.

to the DSD and conventional trial restorations (Figure 7C). The shape of the teeth, length, prominence and line angles were evaluated in addition to the subjective evaluation.

After that, the teeth were minimally prepared, and a definitive impression was made. A second printed cast was used from the DSD for the provisional restoration, representing the final result. The patient was satisfied, but we still tried the 


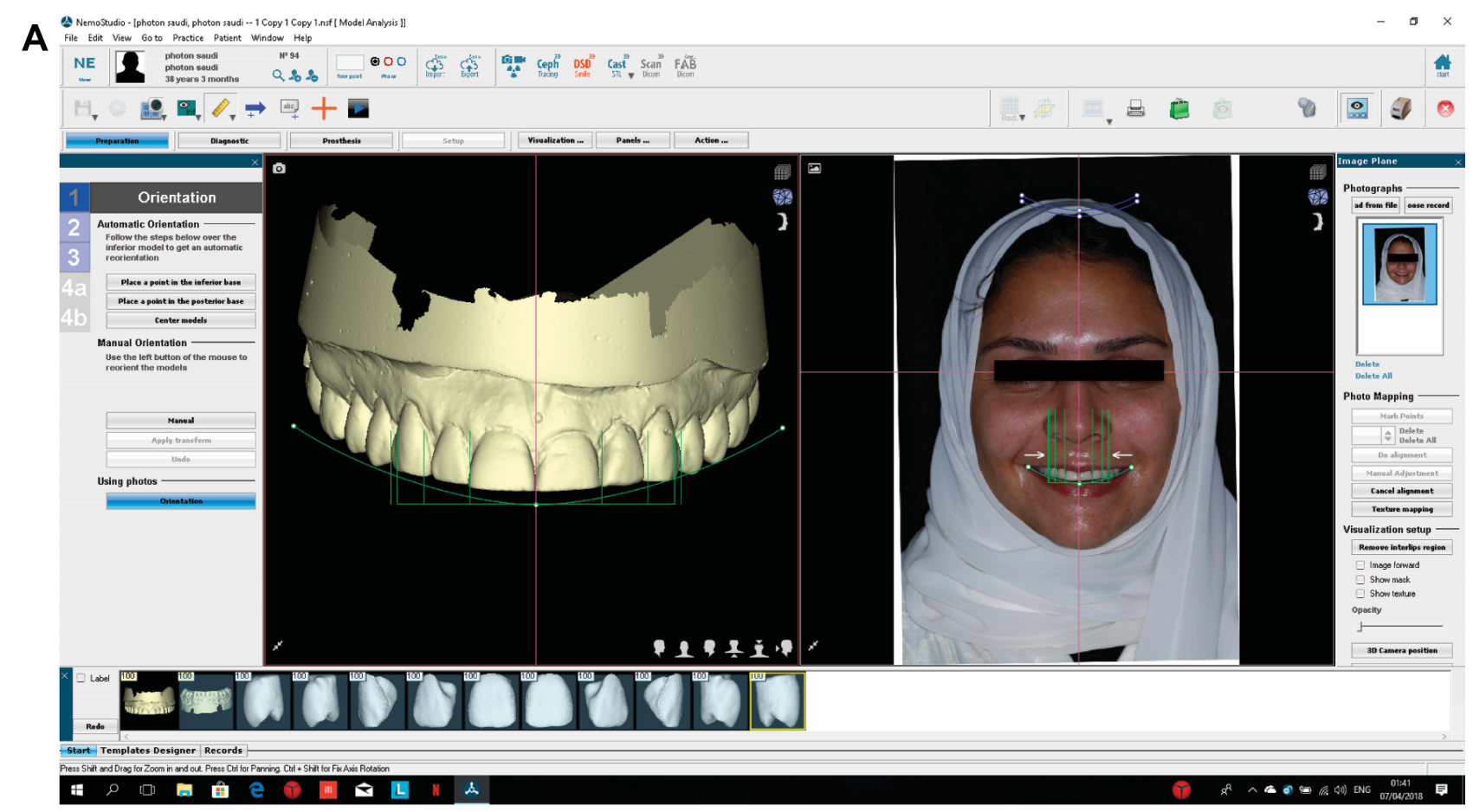

B

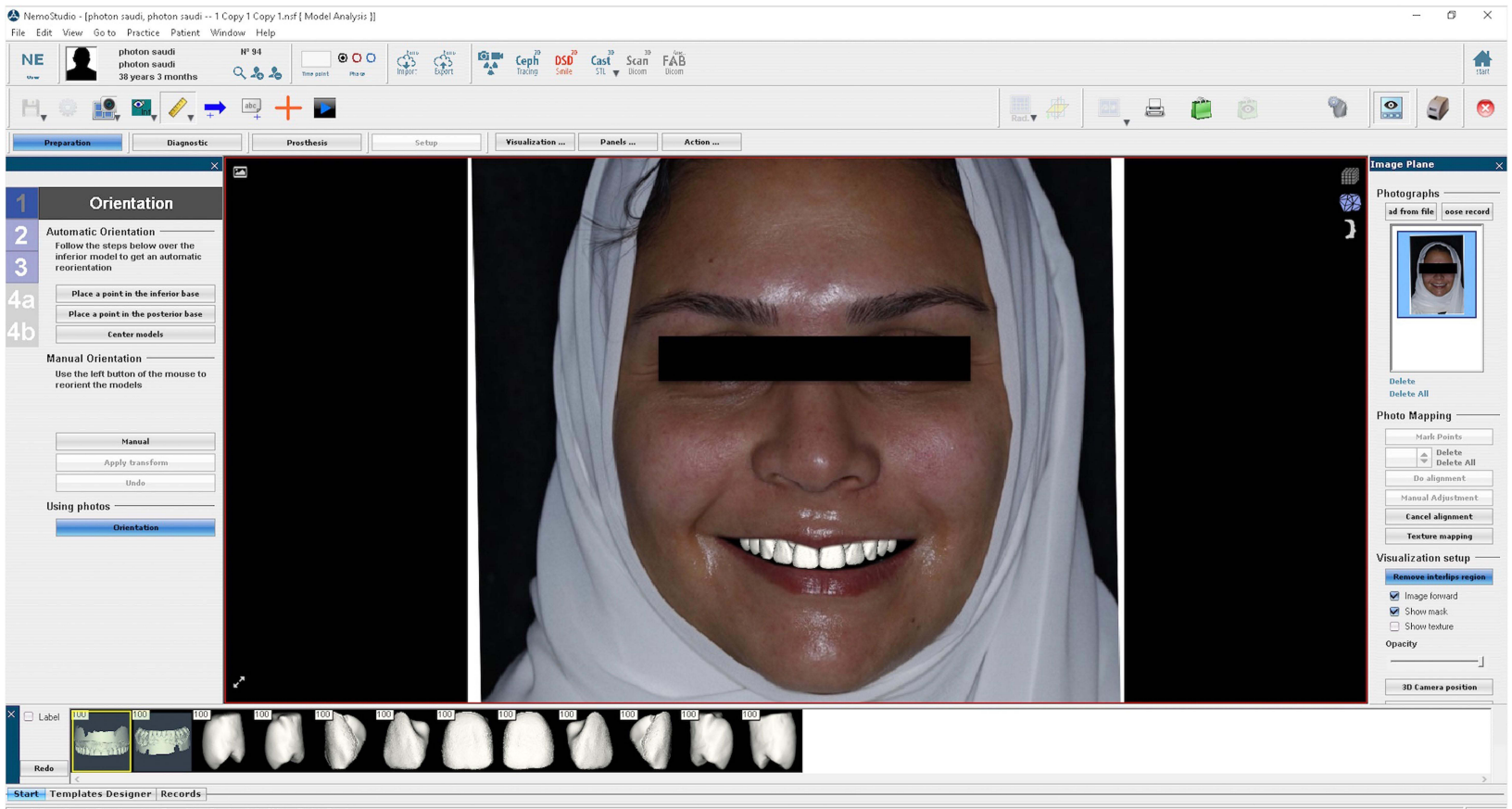

A 0 만

Figure 5 (A) Nemo Digital Smile Design 3D system (DSD) workflow. Matching the cast and the image was done by using two vertical lines on the distal aspects of the canines. (B) Nemo Digital Smile Design 3D system (DSD) digital mock-up.

conventional wax-up as a provisional restoration for comparison and decision making. It was clinically clear that the teeth would need extensive reduction that would reduce the amount of remaining enamel on the prepared tooth structure to accommodate the GPS provisional restoration, so this direction was discontinued. As mentioned before the GPS is a 2D smile design software and the transfer of the data from virtual design to the stone model is susceptible to human errors which might explain the insufficient thickness of the restorations using the GDS index. The final impression was poured twice. The first cast was scanned, and its stereolithography (STL) file and the DSD printed cast were imported 


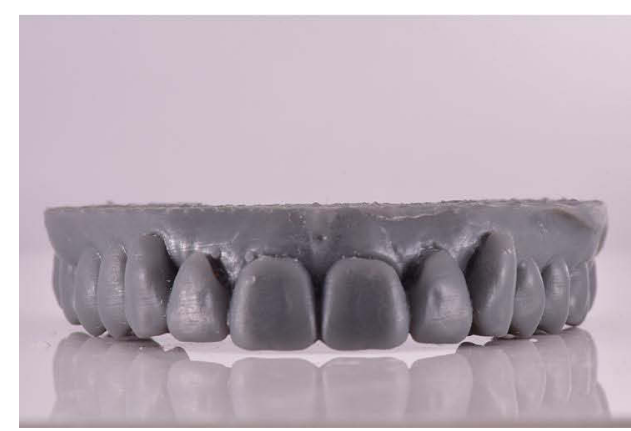

A

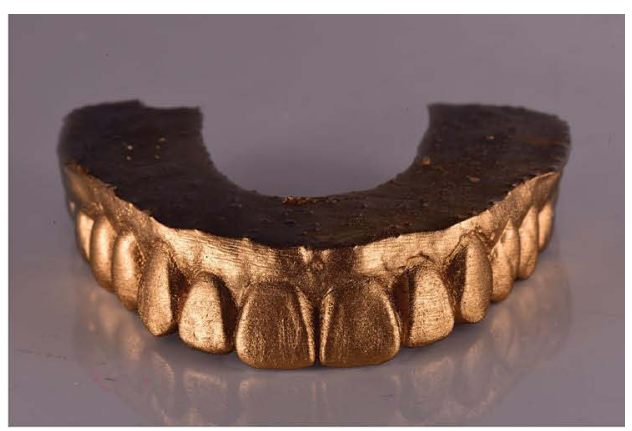

B

Figure 6 (A) Printed cast for mock-up, followed the most protruded central incisor. (B) Printed cast for provisional restoration, mimicking the final design.

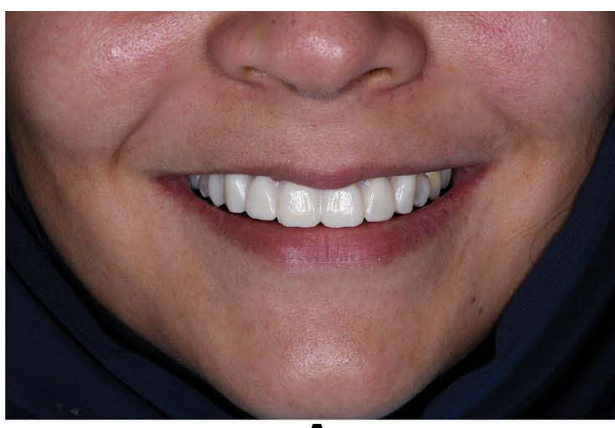

A

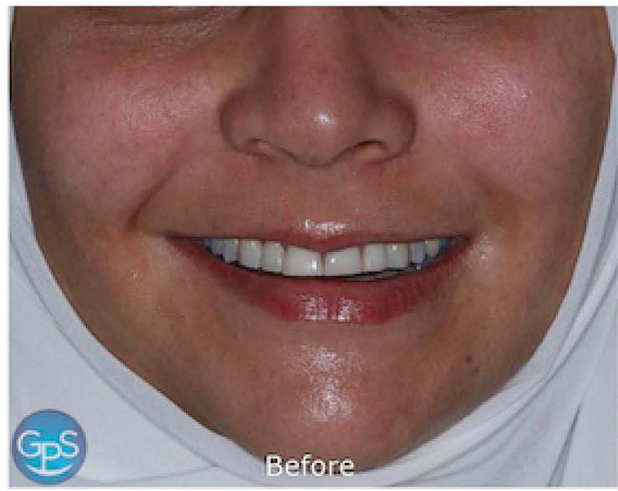

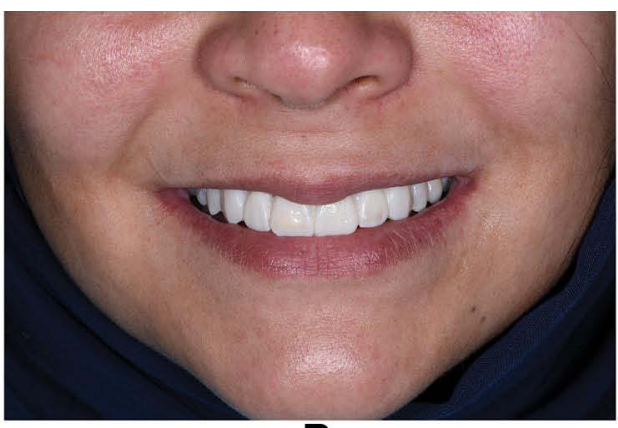

B

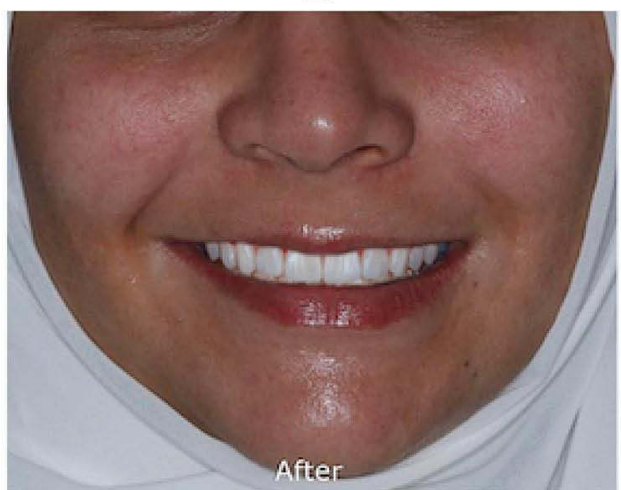

C

Figure 7 (A) Nemo Digital Smile Design 3D system (DSD) mock-up. (B) Conventional workflow mock-up. (C) Guided Positioning System (GPS) mock-up.

and merged in the CAD software. CAM monolithic lithium disilicate ceramic veneers (IPS e.Max CAD LT BL3; Ivoclar Vivadent AG, Schaan, Liechtenstein) were milled and finished with external staining and glazing. The second cast was prepared for individual refractory dies, and feldspathic porcelain was applied using a layering technique and then fired. The silicone index constructed from the wax-up was used as a reference.

On the day of cementation, two sets of veneers were ready to be tried intraorally, milled e.Max veneers (Figure 8A) and feldspathic veneers (Figure 8B), letting the patient make her choice. She chose the feldspathic porcelain veneers for their translucency and shape. Restorations were cemented using light-polymerizing resin cement (Variolink Veneer LC; Ivoclar Vivadent AG), resulting in a satisfactory esthetic outcome then a nightguard was delivered. Postoperative radiographs were taken (Figure 9). 


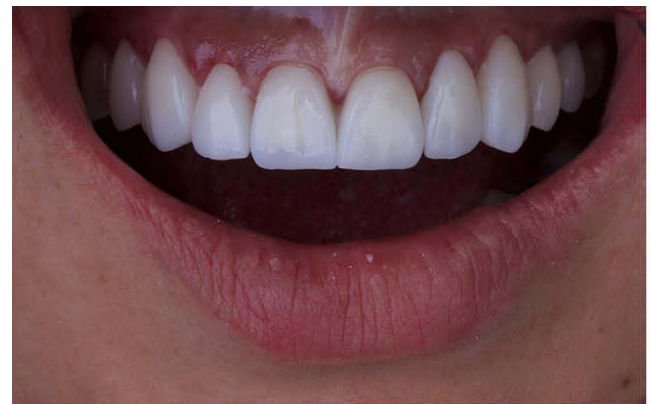

A

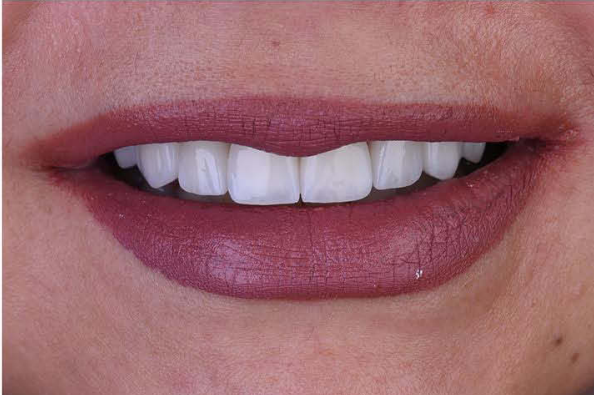

B

Figure 8 (A) Milled e.Max veneers try-in. (B) Feldspathic porcelain veneers after final cementation.
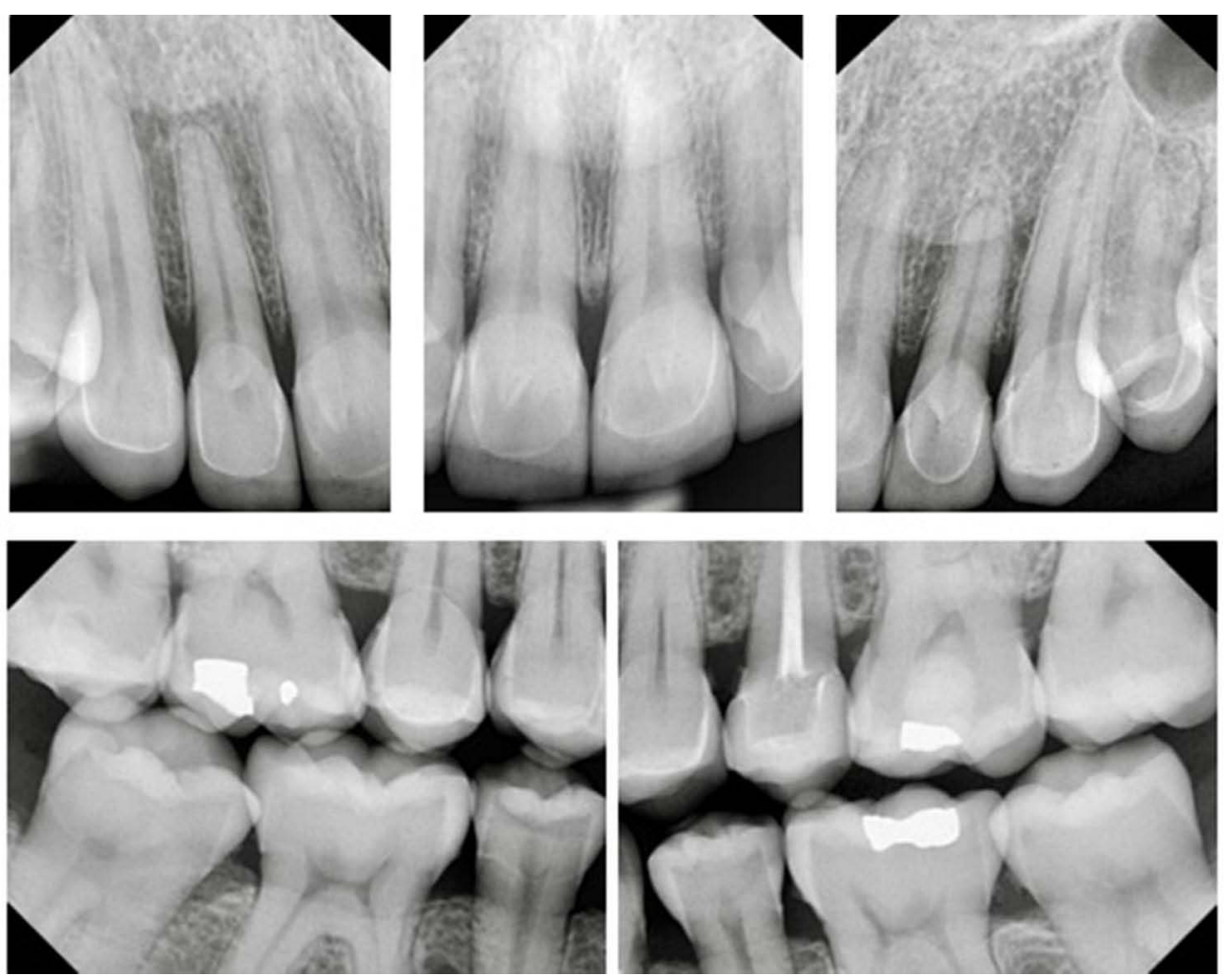

Figure 9 Postoperative radiographs.

\section{Discussion}

In all prosthodontic esthetic treatment, precise diagnosis, comprehensive plan and the communication with the patient play crucial role in predicting the results. ${ }^{6,7}$

Planning for esthetically demanding cases has developed from a wax-up diagnostic stone casts to a virtual design created on a digital photograph with the help of lines and rulers. ${ }^{6,7}$ Successful previsualization and mock-up are very efficient to explain modifications to the patients and get their approval.

Conventional technique is based on a planning process after radiological and clinical examination, intra- and extraoral analysis, occlusal evaluation and impressions. ${ }^{7,8}$ This technique depends mainly on the technician's ability to follow the guideline, hand skills and experience. However, it has a higher chance for human errors. ${ }^{7,8}$ A digital smile design protocol can be limited to digital planning and digital mock-up, like the GPS, which is user friendly and cost-effective 2D software. Various photographic editing instruments are available so the operator can close spaces, graft segments of tooth structure, adjust the smile line, and change the teeth shade within the photo. After digital smile designing, manual 
processing of a laboratory diagnostic wax-up on a calibrated stone model will be completed. But the transfer of the data from virtual design to laboratory stone model is difficult and susceptible to errors. ${ }^{7}$

Nemo DSD 3D system is a minimally invasive totally digitalized process, from the preoperative photo to CAD/CAMprinted mockup. This drastically reduces operator error that usually associated with the conventional manual steps and it improves the accuracy and reproducibility of the prosthetic procedure. ${ }^{7}$ All data transfer from the 3D planning to the laboratory $\mathrm{CAD} / \mathrm{CAM}$ process is faster, easier and more predictable with reduced discomfort and enhanced final esthetic results. $^{7}$

However, in this particular case, we and the patient agreed that the conventional technique gave better results. This can be due to the technician skills was evident in the wax-up and the final restorations. The optical properties of the feldspathic porcelain in compared with the milled Emax can be another reason. Added to this, in this case there is no dark staining or discoloration of the teeth, which might require a more aggressive teeth preparation and/or a higher opacity material. ${ }^{11}$

\section{Summary}

In a normal situation without any discoloration or endodontically treated teeth, it can be concluded that the digital smile design programs demonstrated an effective and predictable workflow, leading to adequate esthetic results. However, the conventional workflow gave conservative and esthetically more pleasing results. The feldspathic veneers were chosen for their superior optical properties. Case complexity and operator experience may have played a significant role in choosing between conventional and digital workflows.

\section{Funding}

The study did not receive any specific grant from funding agencies in the public, commercial, or not-for-profit sectors.

\section{Disclosure}

The authors report no conflicts of interest for this work.

\section{References}

1. Gürel G. Improving the esthetics of healthy teeth with porcelain laminate veneers. In: Cohen M, editor. Interdisciplinary Treatment Planning: Principles, Design, Implementation. Hanover Park: Quintessence Publishing Co, Inc.; 2008:461-502.

2. Coachman C, Calamita M. Digital smile design: a tool for treatment planning and communication in esthetic dentistry. Quintessence Dent Technol. 2012;35:103-111.

3. Omar D, Duarte C. The application of parameters for comprehensive smile esthetics by digital smile design programs: a review of literature. Saudi Dent J. 2018;30(1):7-12. doi:10.1016/j.sdentj.2017.09.001

4. Lin WS, Harris BT, Phasuk K, Llop DR, Morton D. Integrating a facial scan, virtual smile design, and 3D virtual patient for treatment with CADCAM ceramic veneers: a clinical report. J Prosthet Dent. 2018;119:200-205. doi:10.1016/j.prosdent.2017.03.007

5. Pimentel W, Teixeira ML, Costa PP, Jorge MZ, Tiossi R. Predictable outcomes with porcelain laminate veneers: a clinical report. J Prosthodont. 2016;25(4):335-340. doi:10.1111/jopr.12413

6. McLaren EA. Bonded functional esthetic prototype: an alternative pre-treatment mock-up technique and cost-effective medium-term esthetic solution. Compend Contin Educ Dent. 2013;34:596-607.

7. Cattoni F, Mastrangelo F, Gherlone EF, Gastaldi G. A new total digital smile planning technique (3D-DSP) to fabricate CAD-CAM mockups for esthetic crowns and veneers. Int J Dent. 2016;2016:6282587. doi:10.1155/2016/6282587

8. Gurrea J, Bruguera A. Wax-up and mock-up. A guide for anterior periodontal and restorative treatments. Int J Esthet Dent. 2014;9:146-162.

9. De Almeida BA, de Oliveira KF, Caldas RA. Mechanical and optical properties of feldspathic ceramics and lithium disilicate: literature review. Rev Bras Odontol. 2020;77:e1427.

10. Valizadeh S, Ranjbar Omrani L, Deliperi S, Sadeghi Mahounak F. Restoration of a nonvital tooth with fiber reinforce composite (wallpapering technique). Case Rep Dent. 2020;2020:1-6. doi:10.1155/2020/9619787

11. Kermanshah H, Valizadeh S. A 7-year follow-up of a fractured endodontically treated incisor restored with a one-piece post and core laminate veneer. Front Dent. 2019;16(3):224. 
Clinical, Cosmetic and Investigational Dentistry

Dovepress

\section{Publish your work in this journal}

Clinical, Cosmetic and Investigational Dentistry is an international, peer-reviewed, open access, online journal focusing on the latest clinical and experimental research in dentistry with specific emphasis on cosmetic interventions. Innovative developments in dental materials, techniques and devices that improve outcomes and patient satisfaction and preference will be highlighted. The manuscript management system is completely online and includes a very quick and fair peer-review system, which is all easy to use. Visit http://www.dovepress.com/ testimonials.php to read real quotes from published authors.

Submit your manuscript here: https://www.dovepress.com/clinical-cosmetic-and-investigational-dentistry-journal 